\title{
CORRIGENDUM
}

\section{Asymmetric total syntheses of (+)- and (-)-versicolamide B and biosynthetic implications}

\author{
Kenneth A. Miller, Sachiko Tsukamoto and Robert M. Williams
}

Nature Chemistry 1, 63-68 (2009); published online 19 March 2009; corrected after print 6 April 2009.

In the version of this Article originally published, the stereochemical descriptors given in the Methods section for compounds 23, 25, 27 and 29 were incorrect; they have now been corrected in the HTML and PDF versions, and should have appeared as shown below.

23 (S)-3-(((S)-7,7-Dimethyl-3-(2-methylbut-3-en-2-yl)-2-oxo-1,2,3,7-tetrahydropyrano[2,3-g]indol-3-yl)methyl)-2,3,6,7-tetrahydropyrrolo[1,2-a]pyrazine-1,4-dione

25 (S)-3-(((R)-7,7-Dimethyl-3-(2-methylbut-3-en-2-yl)-2-oxo-1,2,3,7-tetrahydropyrano[2,3-g]indol-3-yl)methyl)-2,3,6,7-tetrahydropyrrolo[1,2-a]pyrazine-1,4-dione

27 (R)-3-(((S)-7,7-Dimethyl-3-(2-methylbut-3-en-2-yl)-2-oxo-1,2,3,7-tetrahydropyrano[2,3-g]indol-3-yl)methyl)-2,3,6,7-tetrahydropyrrolo[1,2-a]pyrazine-1,4-dione

29 (R)-3-(((R)-7,7-Dimethyl-3-(2-methylbut-3-en-2-yl)-2-oxo-1,2,3,7-tetrahydropyrano[2,3-g]indol-3-yl)methyl)-2,3,6,7-tetrahydropyrrolo[1,2-a]pyrazine-1,4-dione

An in-house error meant the graphical abstract on the Table of Contents page was missing a double bond in (+)-versicolamide B; this has now been corrected in the HTML and PDF versions, and should have appeared as shown below:

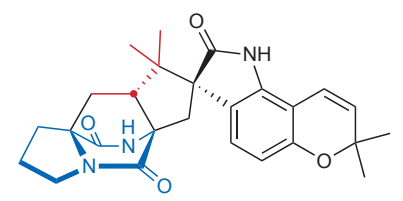

(+)-Versicolamide B

\section{CORRIGENDUM}

\section{Polymer nanofibre junctions of attolitre volume serve as zeptomole-scale chemical reactors}

Pavel Anzenbacher, Jr \& Manuel A. Palacios

Nature Chemistry 1, 82-88 (2009); published online 8 March 2009; corrected after print 16 and 27 March 2009.

In the version of this Article originally published, in paragraph six of the main text, the concentration of reagents in the fibre after evaporation of the solvent was incorrectly given as $0.5 \mathrm{~mol} \mathrm{l}^{-1}$, this should have read $0.5 \mathrm{mmol} \mathrm{l}^{-1}$. The structure given for rhodamine $6 \mathrm{G}$ (compound 5) in Fig. 3a was missing two methyl groups, and should have appeared as shown below. Both these errors have been corrected in the HTML and PDF versions of the Article.<smiles></smiles> 